\title{
Evaluation of 3-D Printed Immobilisation Shells for Head and Neck IMRT
}

\author{
Mark Fisher ${ }^{1}{ }^{*}$, Christopher Applegate ${ }^{1}$, Mohammad Ryalat ${ }^{1}$, Stephen Laycock ${ }^{1}$, \\ Mark Hulse'2, Daniel Emmens ${ }^{3}$, Duncan Bell ${ }^{1,2}$ \\ ${ }^{1}$ School of Computing Sciences University of East Anglia, Norwich Research Park, Norwich, UK \\ ${ }^{2}$ Faculty of Health and Science, University Campus Suffolk, Ipswich, UK \\ ${ }^{3}$ Department of Clinical Oncology, Ipswich Hospital NHS Trust, Ipswich, UK \\ Email: * Mark.Fisher@uea.ac.uk, C.Applegate@uea.ac.uk, M.Ryalat@uea.ac.uk, s.laycock@uea.ac.uk, \\ m.hulse@ucs.ac.uk, Daniel.Emmens@ipswichhospital.nhs.uk, Duncan@fisherideas.co.uk
}

Received 10 October 2014; revised 2 November 2014; accepted 19 November 2014

Copyright (C) 2014 by authors and Scientific Research Publishing Inc.

This work is licensed under the Creative Commons Attribution International License (CC BY).

http://creativecommons.org/licenses/by/4.0/

cC) (i) Open Access

\section{Abstract}

This paper presents the preclinical evaluation of a novel immobilization system for patients undergoing external beam radiation treatment of head and neck tumors. An immobilization mask is manufactured directly from a 3-D model, built using the CT data routinely acquired for treatment planning so there is no need to take plaster of Paris moulds. Research suggests that many patients find the mould room visit distressing and so rapid prototyping could potentially improve the overall patient experience. Evaluation of a computer model of the immobilization system using an anthropomorphic phantom shows that $>99 \%$ of vertices are within a tolerance of $\pm 0.2 \mathrm{~mm}$. Hausdorff distance was used to analyze CT slices obtained by rescanning the phantom with a printed mask in position. These results show that for $\mathbf{8 0} \%$ of the slices the median "worse-case" tolerance is approximately $4 \mathrm{~mm}$. These measurements suggest that printed masks can achieve similar levels of immobilization to those of systems currently in clinical use.

\section{Keywords}

Intensity Modulated Radiotherapy Treatment (IMRT), Patient Immobilization System, 3-D Printing

\section{Introduction}

Before radiation therapy treatment, the dose is prescribed and planned by a team of radiographers, physicists and oncologists using CT imagery. Recommendations regarding the prescription, recording and reporting of external

*Corresponding author.

How to cite this paper: Fisher, M., Applegate, C., Ryalat, M., Laycock, S., Hulse, M., Emmens, D. and Bell, D. (2014) Evaluation of 3-D Printed Immobilisation Shells for Head and Neck IMRT. Open Journal of Radiology, 4, 322-328. 
beam radiation are published in International Commission on Radiation Units and Measurements (ICRU) reports 50 and 62 [1]. The ICRU recommendations make allowances for geometrical uncertainties arising due to variations in patient set-up, organ motion and deformation, and machine related errors that may compromise the exact delivery of the treatment plan [2]. A course of radiation treatment is usually delivered in fractions and may extend over several weeks so unavoidable session-to-session (inter-fraction) variations are managed by adding margins to the treatment volume. Immobilization strategies play an important role in reducing margins and improving treatment outcomes for patients with small lesions adjacent to critical organs such as those undergoing treatment for cancers affecting the brain, head and neck. Immobilization of these patients is normally achieved using an immobilization mask. Typical examples of commonly used immobilization systems are shown in Figure 1.

These are constructed either by directly draping heated thermoplastic over the patient's face or by taking a plaster of Paris negative impression that is subsequently used to create a positive from which a polyethylene immobilization cast is vacuum formed. In either case, the process, which may need to be repeated to ensure that immobilization remains robust as treatment progresses, can be particularly distressing for patients. The Macmillan website describes the plaster of Paris process as potentially uncomfortable [3], but in feedback questionnaires patients have described the process as horrific. Constructing masks is a skilled and time-consuming process that can delay the start of treatment. With recent developments in 3-D printing technologies the potential to generate an immobilization mask directly from a 3-D computer model of a patient comes. Typically, a patient requiring radiation therapy treatment will have had a CT scan and if a computer model of a mask could be obtained directly from the CT data it would reduce patient distress, reduce visits and possibly enable the patient to start their radiation therapy treatment sooner. A number of immobilization mask systems have been developed and technical specifications and methods of implementation vary considerably. High melting point thermoplastic systems prohibit target dislocations larger than $2-3 \mathrm{~mm}$ while lower melting point plastic meshes achieve setup accuracies of between $4-5.5 \mathrm{~mm} \mathrm{[4]} \mathrm{[5].} \mathrm{A} \mathrm{margin} \mathrm{of} 3-4 \mathrm{~mm}$ is typically used when planning radiation therapy treatment to the head and neck to account for this uncertainty [6].

Immobilization systems for head and neck radiotherapy must provide an effective degree of rigidity, together with an acceptable degree of comfort for the patient. An early comparison of different thermoplastic immobilization systems [7] [8] used portal images to assess displacements. These studies concluded that all the systems provided accurate patient immobilization but displacements at the shoulder region were improved by additional fixation points resulting in $90 \%$ of shoulder displacements below $5 \times 5 \mathrm{~mm}$. Nutting et al. [6] review three immobilization strategies for head and neck radiotherapy and conclude that while stereotactic frames similar to those used in brain tumor immobilization are the ultimate immobilization option, custom made Cabulite (cellulose acetate) masks formed over a plaster of Paris impression taken of the patient are more popular. Work evaluating immobilization strategies is ongoing and recent studies using 3-D cone beam CT to assess both translational and rotational set-up errors highlight different rotational accuracies that are hitherto difficult to quantify [9].

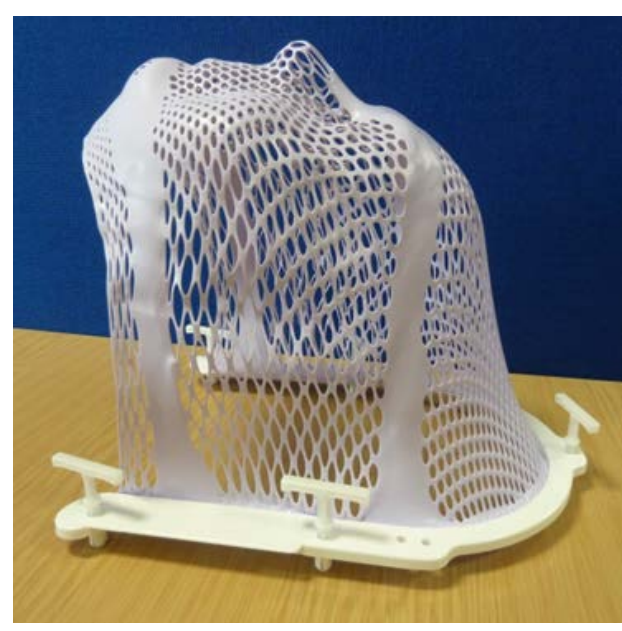

(a)

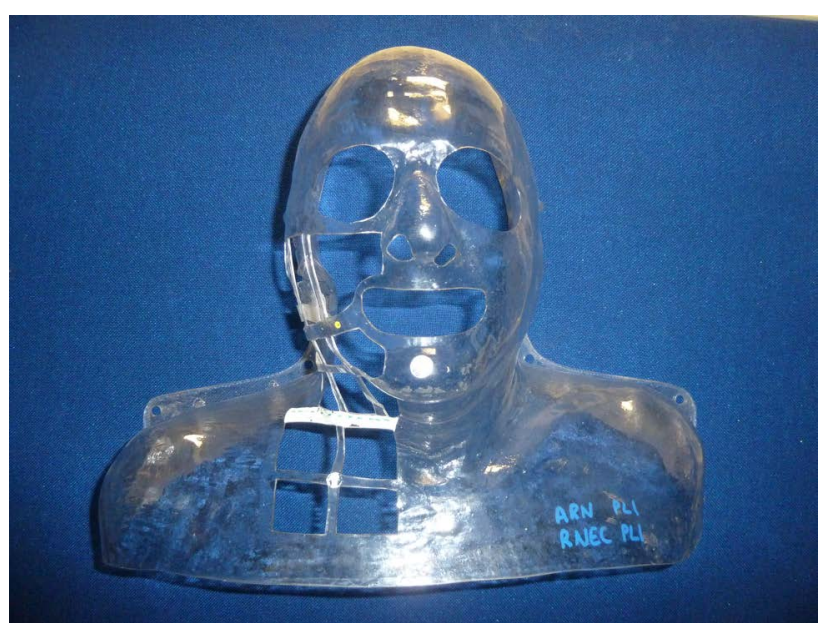

(b)

Figure 1. (a) Thermoplastic; (b) Polyethylene immobilisation masks. 
Technological advances in rapid prototyping and computer aided manufacturing have brought the potential for fabrication of 3-D models to assist in surgical planning and simulation, implantology, neurosurgery, orthopedics and maxillofacial surgery [10]. Rapid manufacturing processes have also been explored as a means of sculpting patient specific shields and masks for shielding and immobilization of patients during radiation therapy treatment [11]-[13]. In addition to improving the quality of treatment, the authors highlight several practical advantages of this approach. In particular, hospitals and treatment facilities no longer need their own manufacturing facilities and manufacturing can be undertaken centrally with potential benefits in terms of cost and accuracy. This paper describes some of the challenges in fabricating printed immobilization masks and assesses quality of fit by measuring the gap between the outer surface of a phantom and the inner surface of the mask. The paper proceeds as follows. First we describe the materials used and the software application we have developed to create physical models of treatment shells directly from the CT data. Then we describe the evaluation strategy, present results and draw conclusions.

\section{Materials}

A diagnostic head phantom was used in the preclinical study. The components are made from tissue simulating resins that mimic X-ray attenuation properties of human tissues. The phantom detail includes 3-D anthropomorphic anatomy comprising brain, bone, larynx, trachea, sinus, nasal cavities and teeth (Figure 2).

CT data sets were acquired using a GE Medical Systems Optima CT scanner with $0.625 \mathrm{~mm}$ inter-slice separation. The masks were created using a Formiga P110 printer with PA2200 material. This printer is manufactured by EOS GmbH and PA2200 is a plastic certified for use in clinical environments, making it particularly appropriate for the construction of immobilization devices which will be in contact with the patients' skin. Earlier work established the X-ray absorption coefficient of this material and confirmed that it would be suitable for use during radiotherapy treatment [13].

\section{Method}

A CT data set $(512 \times 512 \times 409)$ of the phantom was acquired at Ipswich Hospital. A graphics application was written to load DICOM files. Attenuation within each voxel is represented by its CT number

$$
\text { CT number }=1000 \times\left[\left(\mu-\mu_{w}\right) / \mu_{w}\right]
$$

where $\mu$ and $\mu_{w}$ represent the linear attenuation coefficients of the material and water respectively. CT slices were segmented using a threshold (set interactively); flood-filled, dilated using a disk structuring element and an opening is created at the rear (Figure 3 Top Row). The surface contours are represented by smooth parametric curves which can be resampled (as necessary) before rendering as a polygonal mesh and exported in Stereolithography (STL) file format for printing (Figure 3 Bottom Row).

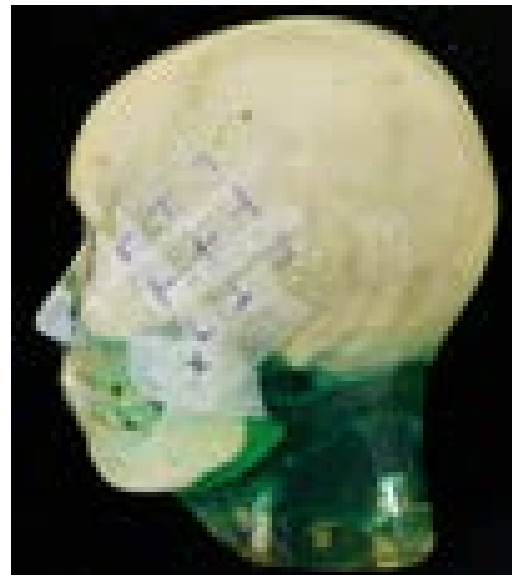

Left

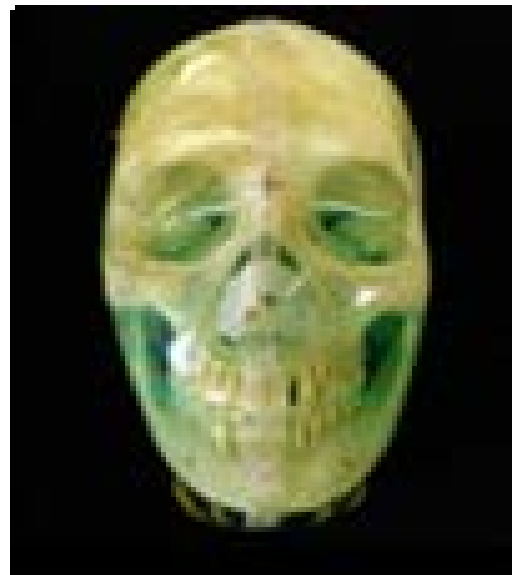

Front

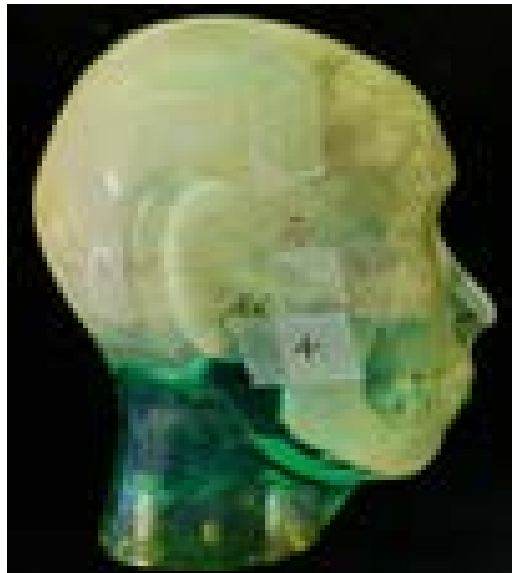

Right

Figure 2. Anthropomorphic phantom ("Eddie”). 

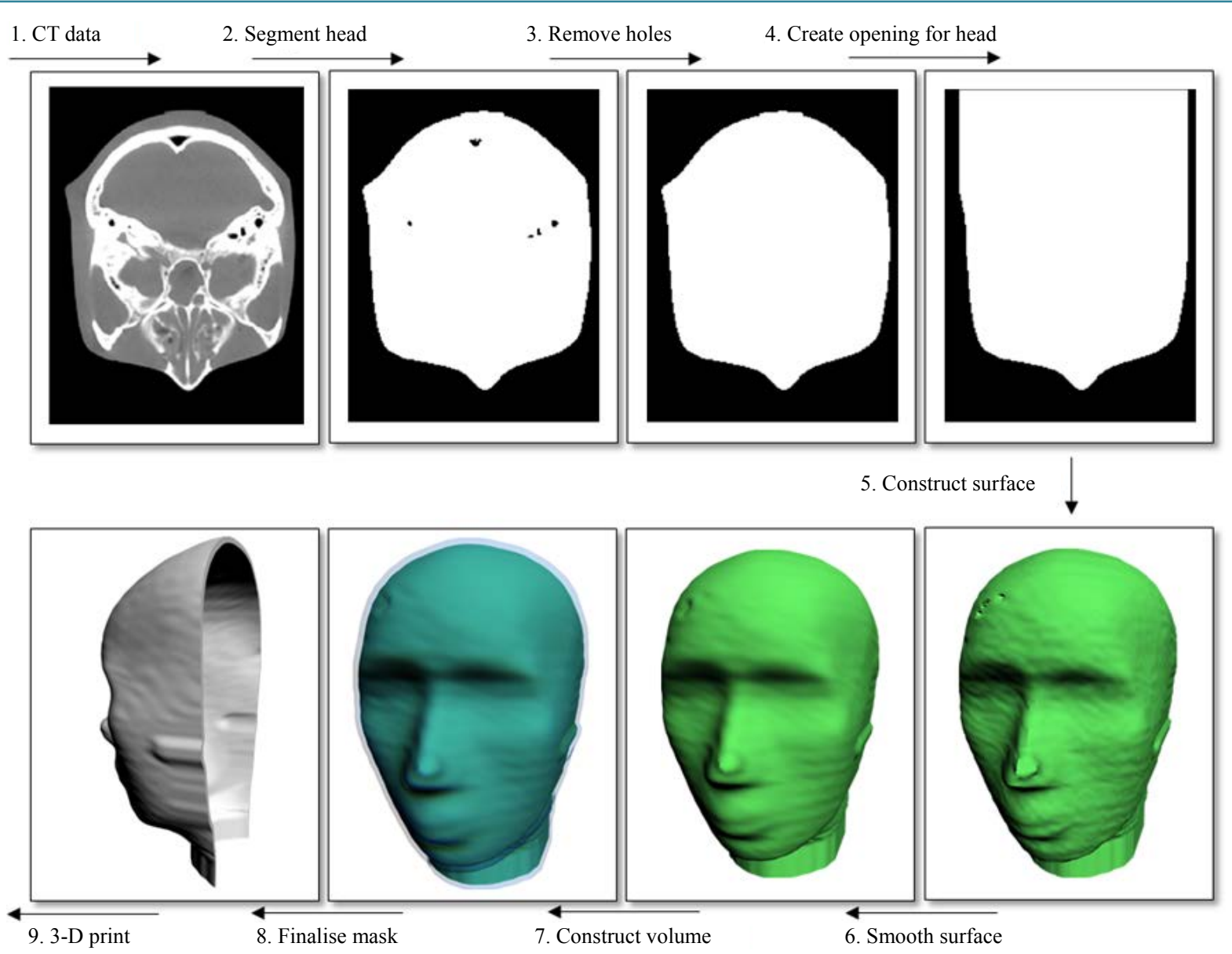

Figure 3. Mask generation processing pipeline: Top Row—Slice related image processing; Bottom Row_-3D reconstruction.

\section{Experimental Results}

To evaluate the accuracy of the modelling process and to measure the variability in mask thickness, we have developed an error analysis tool. The tool renders the distance between the 3-D model of the patient's head and the inner surface of the mask as a colour map (Figure 4). After printing the immobilization mask we rescanned the phantom once with and without the mask placed in position to assess the accuracy of the fabrication process and to evaluate the dilation process used to adjust the fit (Figure 5(a)). The CT slices were converted to binary images by applying a threshold value determined using Otsu's method [14] (Figure 5(b)).

Hausdorff distance

$$
H(A, B)=\max (h(A, B), h(B, A))
$$

where

$$
h(A, B)=\max _{a \in A} \min _{b \in B}\|a-b\|
$$

and $\|\cdot\|$ represents the Euclidean norm, was used to measure the worse-case gap between the skull and mask in binary slices [15]. These measurements are presented in Figure 5(c) and Figure 5(d).

\section{Discussion}

Error analysis carried out on computer models of masks derived from the phantom shows that dilation using a morphological disk structuring element to control the gap delivers acceptable tolerance $(<1 \mathrm{~mm})$. However, comparing values measured by the model evaluation tool and those measured using Hausdorff distance we can 


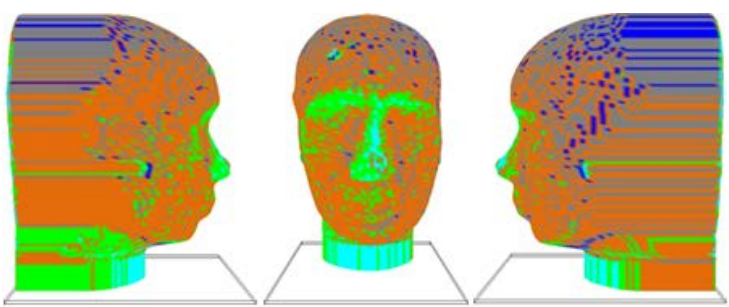

Gap $(1 \mathrm{~mm})$
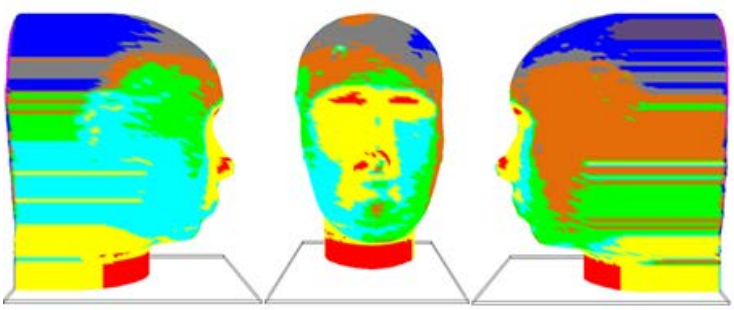

Thickness $(4 \mathrm{~mm})$
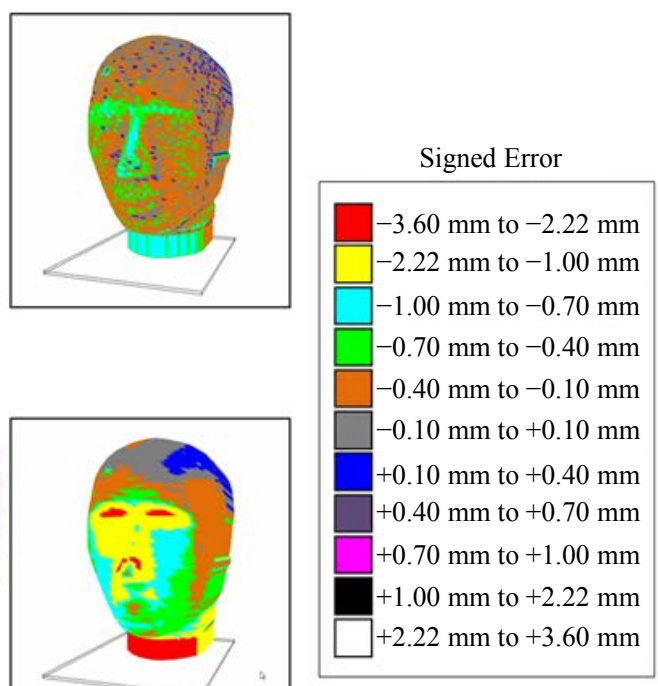

Figure 4. 3-D model evaluation of gap between face and inner surface of mask and thickness of mask. Note: Parameters shown in brackets refer to size of morphological disk used in dilation step.

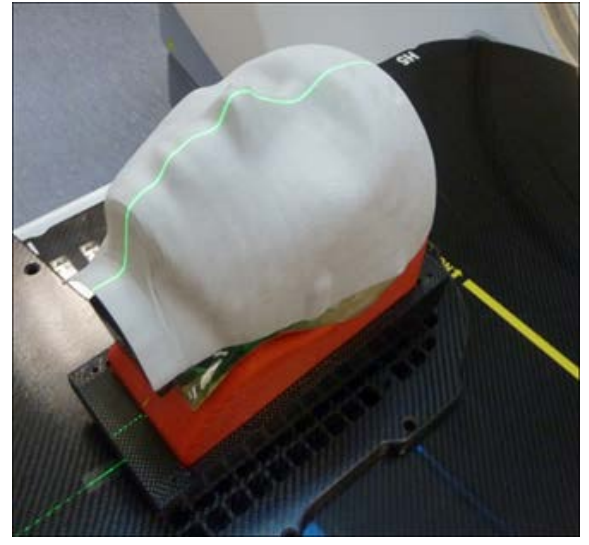

(a)

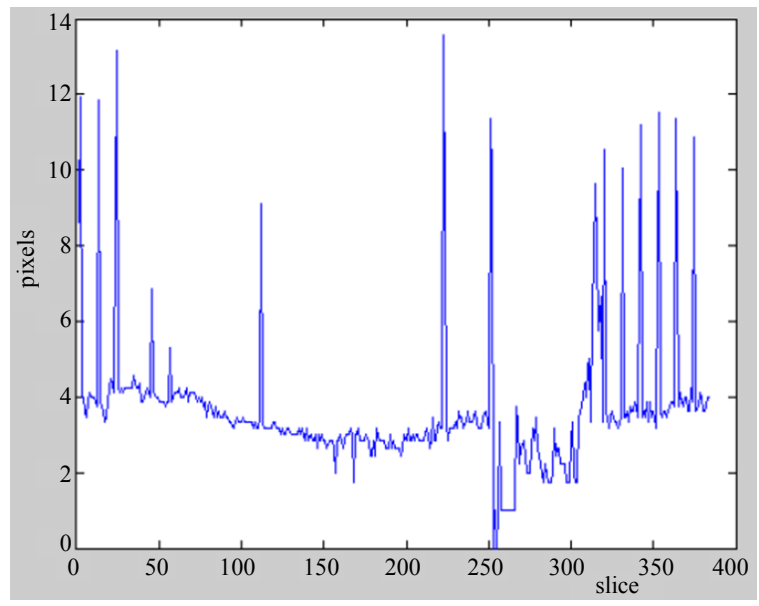

(c)

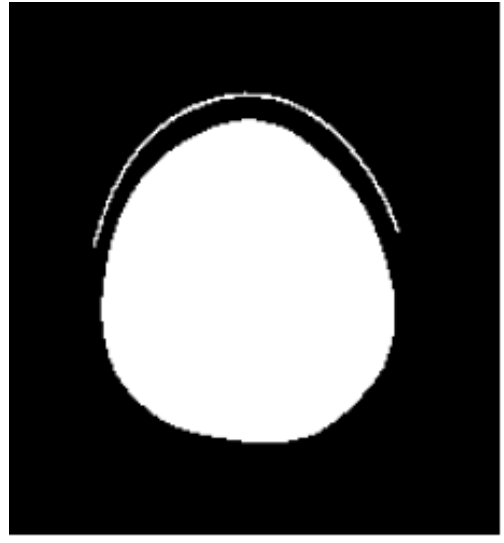

(b)

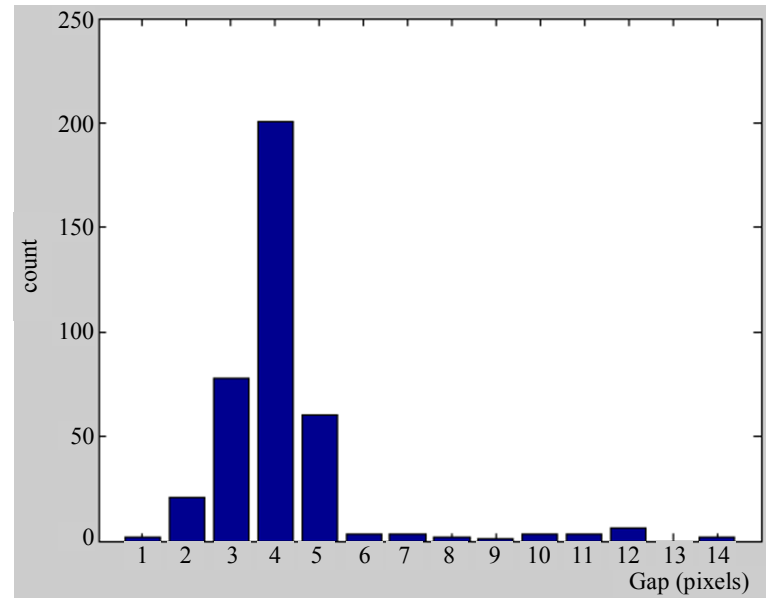

(d)

Figure 5. (a) Rescanned phantom fitted with printed mask; (b) A single (binarised) slice; (c) Slice-by-slice evaluation of fit using Hausdorff distance; (d) Histogram of Hausdorff distance. 
see that although in $>80 \%$ of the slices the largest gap is within $4 \mathrm{~mm}$ it can rise to $14 \mathrm{~mm}$ in a small number of slices. The size of these errors $(14 \mathrm{~mm})$ leads us to suspect they are anomalous values measured at the periphery of the mask and we anticipate they will be eliminated when an interface to a fixation board is incorporated. This feature will enable us to assess the robustness of the system by refitting the printed immobilization mask and rescanning on a number of occasions.

Our initial results suggest that such a system could deliver a degree of immobilization but the difference in accuracy between the model and physical realization is a matter of concern. The most likely cause of this is in the choice of threshold (chosen interactively and by Otsu's technique) used to establish the surface of the skin. We anticipate that this step will be much more challenging in clinical practice due to subcutaneous tissue that is not well determined in CT images. Although MRI is better suited to this task this modality is not routinely available for radiotherapy treatment planning. Other approaches to building 3-D facial models such as laser scanning and photogrammetry also offer potential solutions that could be adopted a relatively low cost.

\section{Conclusion}

This paper demonstrates the feasibility of manufacturing patient specific immobilisation masks using a 3-D printer. This manufacturing technique can potentially reduce the stress and discomfort experienced by patients undergoing radiotherapy to the head and neck. Preclinical trials using a phantom suggest that masks manufactured using CT data suffer inaccuracies due to difficulties in determining a suitable threshold to accurately locate the surface of the face. Although the errors are small (approx. $3-4 \mathrm{~mm}$ ) in the phantom we anticipate that this could be a more serious issue in studies involving human subjects. We are currently investigating other 3-D capture techniques that could be used to improve the accuracy. Other engineering problems associated with the fixation interface need to be addressed before clinical trials are undertaken and once these are complete we expect further results to confirm that the system provides a suitable degree of comfort whilst affording an adequate level of physical immobilization.

\section{Acknowledgements}

This work was funded by Norfolk Cancer Charity Big C grant no. 12-07R: Automatic construction of radiotherapy treatment shells. We would like to thank Garth Stephenson and EOS (http://www.eos.info/en) for their collaboration and for providing free manufacture of prototype shells and Consultant Oncologist Christopher Scrase and Senior Therapeutic Radiographer Suzanne Isherwood at Ipswich Hospital for their help and access to X-ray CT.

\section{References}

[1] ICRU (1999) ICRU Report 62: Prescribing, Recording and Reporting Photon Beam Therapy (Supplement to ICRU Report 50). ICRU, Bethesda.

[2] Stroom, J.C. and Heijmen, B.J. (2002) Geometrical Uncertainties, Radiotherapy Planning Margins, and the ICRU-62 Report. Radiotherapy and Oncology, 64, 75-83. http://dx.doi.org/10.1016/S0167-8140(02)00140-8

[3] Macmillan Cancer Support (2013) Making a Radiotherapy Mask. [Online] http://www.macmillan.org.uk/Cancerinformation/Cancertreatment/Treatmenttypes/Radiotherapy/Beforetreatment/Radi otherapymasks.aspx

[4] Fuss, M., Salter, B.J., Cheek, D., Sadeghi, A., Hevezi, J.M. and Herman, T.S. (2004) Repositioning Accuracy of a Commercially Available Thermoplastic Mask System. Radiotherapy and Oncology, 71, 339-345. http://dx.doi.org/10.1016/j.radonc.2004.03.003

[5] The Royal College of Radiologists, Society and College of Radiographers, Institute of Physics and Engineering in Medicine (2008) On Target: Ensuring Geometric Accuracy in Radiotherapy. The Royal College of Radiologists, London.

[6] Nutting, C., Bidmead, A. and Henk, J. (2003) Geometric Uncertainties in Radiotherapy of Head and Neck Cancer. In: McKenzie, A., Ed., Geometric Uncertainties in Radiotherapy: Defining the Planning Target Volume, The British Institute of Radiology, London, 127-141.

[7] Gilbeau, L., Octave-Prignot, M., Loncol, T., Renard, L., Scalliet, P. and Grégoire, V. (2001) Comparison of Setup Accuracy of Three Different Thermoplastic Masks for the Treatment of Brain and Head and Neck Tumors. Radiotherapy and Oncology, 58, 155-62. http://dx.doi.org/10.1016/S0167-8140(00)00280-2 
[8] Rotondo, R.L., Sultanem, K., Lavoie, I., Skelly, J. and Raymond, L. (2008) Comparison of Repositioning Accuracy of Two Commercially Available Immobilization Systems for Treatment of Head-and-Neck Tumors Using Simulation Computed Tomography Imaging. International Journal of Radiation Oncology, Biology, Physics, 70, 1389-1396.

[9] Christiansen, R., Hansen, C., Nielsen, T., Johansen, J. and Brink, C. (2012) Comparison of Three Immobilisation Systems for Radiation Therapy in Head and Neck Cancer. Radiotherapy \& Oncology, 103, S106. http://dx.doi.org/10.1016/S0167-8140(12)70609-6

[10] Goiato, M., Pesqueira, A. and dos Santos, D. (2011) Prototyping for Surgical and Prosthetic Treatment. The Journal of Craniofacial Surgery, 22, 914-917. http://dx.doi.org/10.1097/SCS.0b013e31820f7f90

[11] McKernan, B., Bydder, S., Deans, T., Nixon, M. and Joseph, D. (2007) Surface Laser Scanning to Routinely Produce Casts for Patient Immobilization during Radiotherapy. Australasian Radiology, 51, 150-153. http://dx.doi.org/10.1111/j.1440-1673.2007.01686.x

[12] de Beer, D.J., Truscott, M., Booysen, G.J., Barnard, L.J. and van der Walt, J.G. (2005) Rapid Manufacturing of Patient-Specific Shielding Masks, Using RP in Parallel with Metal Spraying. Rapid Prototyping Journal, 11, 298-303. http://dx.doi.org/10.1108/13552540510623611

[13] Laycock, S.D., Hulse, M., Scrase, C.D., Tam, M.D., Isherwood, S., Mortimore, D.B., Emmens, D., Patman, J., Short, S.C. and Bell, G.D. (2014) Towards the Production of Radiotherapy Treatment Shells on 3D Printers Using Data Derived from DICOM CT and MRI: Preclinical Feasibility Studies. Journal of Radiotherapy in Practice, 1-7. http://dx.doi.org/10.1017/S1460396914000326

[14] Otsu, N. (1979) A Threshold Selection Method from Gray-Level Histograms. IEEE Transactions on Systems, Man and Cybernetics, 9, 62-66. http://dx.doi.org/10.1109/TSMC.1979.4310076

[15] Huttenlocher, D., Klanderman, G. and Rucklidge, W. (1993) Comparing Images Using the Hausdorff Distance. IEEE Transactions on Pattern Analysis and Machine Intelligence, 15, 850-863. http://dx.doi.org/10.1109/34.232073 
Scientific Research Publishing (SCIRP) is one of the largest Open Access journal publishers. It is currently publishing more than 200 open access, online, peer-reviewed journals covering a wide range of academic disciplines. SCIRP serves the worldwide academic communities and contributes to the progress and application of science with its publication.

Other selected journals from SCIRP are listed as below. Submit your manuscript to us via either submit@scirp.org or Online Submission Portal.
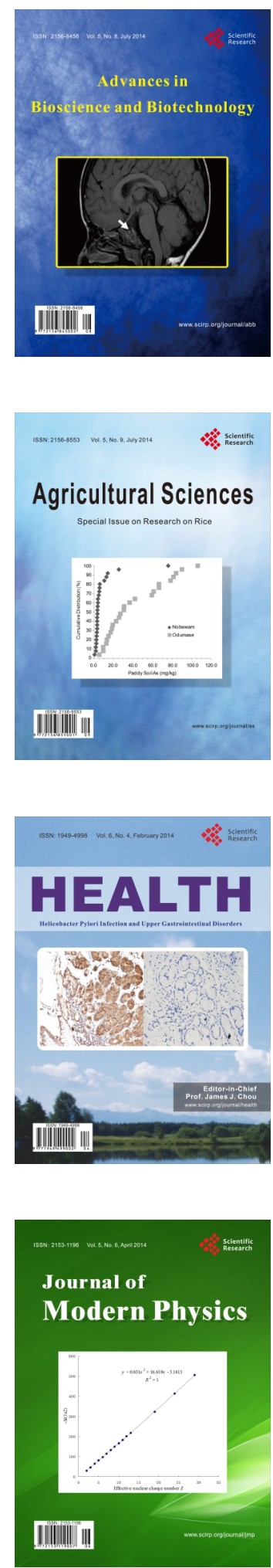
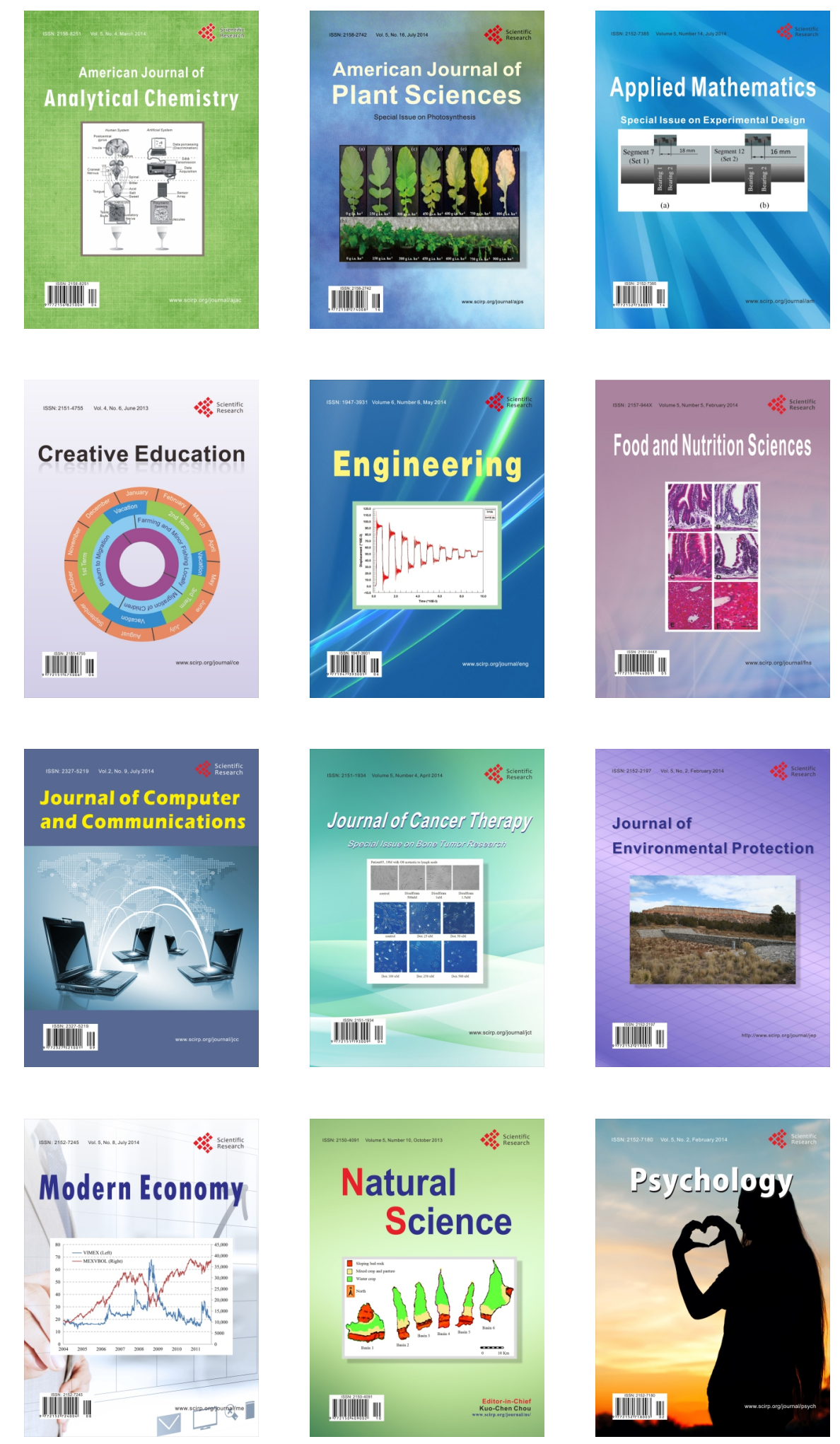